\title{
THE NULL-CONE AND COHOMOLOGY OF VECTOR BUNDLES ON FLAG VARIETIES
}

\author{
KARI VILONEN AND TING XUE
}

\begin{abstract}
We study the null-cone of a semi-simple algebraic group acting on a number of copies of its Lie algebra via the diagonal adjoint action. We show that the null-cone has rational singularities in the case of $S L_{3}$. We observe by example that the null-cone is not normal in general and that the normalization of the null-cone does not have rational singularities in general. This is achieved by computing cohomology of certain vector bundles on flag varieties.
\end{abstract}

\section{INTRODUCTION}

In this paper we study the null-cone of a semi-simple algebraic group acting on a number of copies of its Lie algebra via the diagonal adjoint action. Such actions and their generalizations have been considered by various authors; see, for example, [CM, KW1, KW2,LMP. Our interest in the question was motivated by applications in the study of ordinary deformations of Galois representations. This point of view is due to Snowden who studied the case $\mathfrak{g}=\mathfrak{s l}_{2}$ in [S]. In the case of $\mathfrak{g}=\mathfrak{s l}_{2}$ he shows that the null-cones (and other related spaces that come up in ordinary deformations of Galois representations) are Cohen-Macaulay but not Gorenstein. In the case of characteristic zero the method of Snowden amounts to proving that the null-cone has rational singularities. In this paper we show that for $\mathfrak{g}=\mathfrak{s l}_{3}$ the null-cones do still have rational singularities, and hence are Cohen-Macaulay. However, we also show that this fails in general. For example, it is not difficult to see that the null-cone is not normal when the group is of type $B_{2}$. In type $A_{5}$ we further show that the normalization of the null-cone does not have rational singularities. We do this by giving estimates on cohomology groups of equvariant vector bundles on flag varieties.

In [H] Hesselink shows that the nilpotent cone of a semi-simple Lie algebra has rational singularities. As the cotangent bundle of the flag variety is a resolution of singularities of the nilpotent cone, one is reduced to proving cohomology vanishing of the symmetric algebra of the tangent bundle of the flag variety. By utilizing the Koszul complex this is reduced to the Borel-Weil-Bott theorem. In our case of multiple copies of the Lie algebra the situation is more complicated as one is reduced to analyzing the cohomology of symmetric powers of direct sums of copies of the tangent bundle of a flag variety. This, in turn, is reduced to analyzing

Received by the editors February 3, 2016 and, in revised form, March 10, 2016 and October 14, 2016.

2010 Mathematics Subject Classification. Primary 14L99, 20 G99.

The first author was supported in part by NSF grants DMS-1402928 and DMS-1069316, the Academy of Finland, and the ARC grant DP150103525.

The second author was supported in part by the ARC grants DP150103525, DE160100975, and the Academy of Finland. 
cohomology groups of the whole tensor algebra of the tangent bundle. We carry out this analysis just far enough to produce the counterexamples. The calculation of the cohomology groups of the tensor algebra of the tangent bundle appears to be a difficult problem.

The paper is organized as follows. In section 2 we introduce the null-cone and in section 3 we make some general remarks on cohomology of equivariant vector bundles on flag varieties. In section 4 we reduce the question of rational singularities to a question of cohomology of equivariant vector bundles on flag varieties. In section 5 we compute a large enough piece of the cohomology of certain equivariant vector bundles and in section 6 we state and prove our main results. We manage to perform this computation sufficiently far enough to produce the counterexamples.

As our results in this paper are mostly counterexamples we will just work in characteristic zero and will only comment on the finite characteristic case.

\section{The NULL-CONE}

We work over a field of characteristic zero which we can and will, for simplicity, take to be $\mathbb{C}$. Let $\mathfrak{g}$ be a semi-simple Lie algebra, and let us write $G$ for the corresponding adjoint group $\operatorname{Aut}(\mathfrak{g})^{0}$.

We write $\mathfrak{b}$ for Borel subalgebras of $\mathfrak{g}$ and $\mathfrak{n}=[\mathfrak{b}, \mathfrak{b}]$ for their nil-radicals. Similarly, we write $B$ for Borel subgroups of $G$.

The group $G$ acts diagonally on $\mathfrak{g}^{\oplus r}$ via the adjoint action. We write

$$
\mathcal{A}_{r}=\left\{\left(x_{1}, \ldots, x_{r}\right) \in \mathfrak{g}^{\oplus r} \mid f\left(x_{1}, \ldots, x_{r}\right)=0 \forall f \in \mathbb{C}\left[\mathfrak{g}^{\oplus r}\right]_{+}^{G}\right\}
$$

for the (reduced) invariant theory null-cone associated to this action. The null-cone itself is, in general, a non-reduced scheme, but in what follows we will work with its underlying reduced scheme structure. It is not difficult to see that this variety can also be described in the following manner (see [KW2]):

$$
\mathcal{A}_{r}=\left\{\left(x_{1}, \ldots, x_{r}\right) \in \mathfrak{g}^{\oplus r} \mid \exists \text { Borel subalgebra } \mathfrak{b} \text { such that } x_{i} \in \mathfrak{n} \text { for all } i\right\} .
$$

Furthermore, in the case $\mathfrak{g}=\mathfrak{s l}_{n}$, we can view $\mathcal{A}_{r}$ as

$$
\mathcal{A}_{r}=\left\{\left(x_{1}, \ldots, x_{r}\right) \in \mathfrak{g}^{\oplus r} \mid x_{i_{1}} \cdots x_{i_{n}}=0 \text { for all choices of } i_{1}, \ldots, i_{n}\right\} .
$$

The variety $\mathcal{A}_{r}$ has a natural $G$-equivariant resolution of singularities which can be described as follows. Let $X$ denote the flag variety of $G$, then the resolution is given by

$$
\widetilde{\mathcal{A}}_{r}:=G \times_{B} \mathfrak{n}^{\oplus r} \cong\left(T^{*} X\right)^{\oplus r} \stackrel{\varphi_{r}}{\longrightarrow} \mathcal{A}_{r},
$$

where the map $\varphi_{r}$ is given by $\varphi_{r}\left(g, x_{1}, \ldots, x_{r}\right)=\left(g x_{1}, \ldots, g x_{r}\right)$. We write

$$
\pi: \widetilde{\mathcal{A}}_{r} \cong\left(T^{*} X\right)^{\oplus r} \rightarrow X
$$

for the projection.

In the case of $r=1$ the null-cone $\mathcal{A}_{1}=\mathcal{N}$ is the usual nilpotent cone and the resolution $\varphi_{1}: T^{*} X \rightarrow \mathcal{N}$ is often referred to as the Springer resolution. In this case the null-cone is reduced and the ring of invariants exhibits it as a complete intersection. In the case of $r=2$ Charbonnel and Moreau CM defined a nilpotent bicone and they show that it is a complete intersection and is in general not reduced. The variety $\mathcal{A}_{2}$ is an irreducible component of the bicone.

The case of $\mathfrak{g}=\mathfrak{s l}_{2}$ was studied by Snowden [S] where he proves that in this case $\mathcal{A}_{r}$ has rational singularities and so is, in particular, Cohen-Macaulay. 
As $\varphi_{r}: \widetilde{\mathcal{A}}_{r} \rightarrow \mathcal{A}_{r}$ is a resolution of singularities of an affine variety we conclude that

$$
\Gamma\left(\widetilde{\mathcal{A}}_{r}, \mathcal{O}_{\widetilde{\mathcal{A}}_{r}}\right) \text { is the normalization of } \mathbb{C}\left[\mathcal{A}_{r}\right]
$$

It is easy to see that $\mathcal{A}_{r}$ has rational singularities, i.e., that $R \varphi_{r *} \mathcal{O}_{\widetilde{\mathcal{A}}_{r}} \cong \mathcal{O}_{\mathcal{A}_{r}}$ if and only if

$$
\mathrm{H}^{i}\left(\widetilde{\mathcal{A}}_{r}, \mathcal{O}_{\widetilde{\mathcal{A}}_{r}}\right)=0 \text { for } i>0 \quad \text { and } \quad \Gamma\left(\widetilde{\mathcal{A}}_{r}, \mathcal{O}_{\widetilde{\mathcal{A}}_{r}}\right)=\mathbb{C}\left[\mathcal{A}_{r}\right]
$$

\section{Cohomology of EQuivariant vector Bundles}

Let us consider the flag variety $X=G / B$, where we think of having chosen a particular Borel subgroup $B$ as a base point. Then we have an equivalence of categories:

$$
\{G \text {-equivariant vector bundles on } X\} \longleftrightarrow\{B \text {-representations }\} \text {. }
$$

Given a $B$-representation $E$ we will use the same symbol $E$ to denote the corresponding vector bundle. Thus, $\mathrm{H}^{k}(X, E)$ stands for the cohomology groups of the $G$-equivariant vector bundle associated to $E$. The cohomology groups $\mathrm{H}^{k}(X, E)$ are $G$-representations, of course.

Let us write $T \subset B$ for a maximal torus and $X^{*}(T)$ for the group of characters $\lambda: T \rightarrow \mathbb{G}_{m}$. We also write $\Phi \subset X^{*}(T)$ for the set of roots and we choose a positive root system $\Phi^{+}$such that the roots in $B$ are negative. We also write

$$
X^{+}(T)=\left\{\lambda \in X^{*}(T) \mid\langle\lambda, \check{\alpha}\rangle \geq 0 \text { for } \alpha \in \Phi^{+}\right\}
$$

for the dominant weights; here $\check{\alpha}$ stands for the coroot associated to $\alpha$. We write $L(\lambda)$ for the irreducible representation of $G$ associated to the highest weight $\lambda \in$ $X^{+}(T)$. Given a representation $V$ of $G$ we write

$$
\operatorname{Supp}(V)=\left\{\lambda \in X^{+}(T) \mid L(\lambda) \text { occurs as a direct summand in } V\right\}
$$

for the support of $V$; it is, of course, a subset of the dominant weights $X^{+}(T)$.

We can view each $\lambda \in X^{*}(T)$ also as a character of $B$ and in this manner $\lambda$ gives rise to a $G$-equivariant line bundle $\mathcal{L}_{\lambda}$. If $\lambda$ is dominant, then $L(\lambda)=\mathrm{H}^{0}\left(X, \mathcal{L}_{\lambda}\right)$. The "dot" action of the Weyl group $W$ on $X^{*}(T)$ is given by $w \cdot \lambda=w(\lambda+\rho)-\rho$; here $\rho$, as usual, is half the sum of positive roots. We recall the statement of the Borel-Weil-Bott theorem:

If there exists a $w \in W$ such that $w \cdot \lambda \in X^{+}(T)$, then

$$
\mathrm{H}^{k}\left(X, \mathcal{L}_{\lambda}\right) \simeq \begin{cases}\mathrm{H}^{0}\left(X, \mathcal{L}_{w \cdot \lambda}\right) & \text { if } k=l(w) \\ 0 & \text { otherwise }\end{cases}
$$

This statement says, in particular, that if there is no $w \in W$ such that $w \cdot \lambda$ is dominant, then the cohomology of $\mathcal{L}_{\lambda}$ vanishes in all degrees.

Consider a $B$-representation $E$ and the cohomology groups $\mathrm{H}^{k}(X, E)$ of the corresponding $G$-equivariant vector bundle. We will give a simple upper bound for $\operatorname{Supp}\left(\mathrm{H}^{k}(X, E)\right)$. Let us choose a filtration of the $B$-representation $E$ such that the associated graded gr $E$ is a direct sum of one-dimensional representations, i.e., of characters of $T$. Let us write $\chi(E)$ for the set of characters appearing in this direct sum decomposition:

$$
\operatorname{gr} E \cong \bigoplus_{\lambda_{i} \in \chi(E)} \lambda_{i}^{\oplus n_{i}}
$$


Thus, we obtain a filtration of the $G$-bundle $E$ such that the associated graded is a direct sum of $G$-equivariant line bundles. From this it is easy to conclude:

$$
\operatorname{Supp}\left(\mathrm{H}^{k}(X, E)\right) \subset\left\{\lambda \in X^{+}(T) \mid w \cdot \lambda \in \chi(E) \text { for a } w \in W \text { with } \ell(w)=k\right\} .
$$

Let us call the right-hand side of this equality the potential support of $\mathrm{H}^{k}(X, E)$, and we write $\operatorname{PSupp}\left(\mathrm{H}^{k}(X, E)\right)$ for it.

Let us recall that either by a direct calculation or using the Hodge decomposition for the flag variety and, identifying $\mathfrak{n}$ and $(\mathfrak{g} / \mathfrak{b})^{*}$ via the Killing form, we conclude:

$$
\mathrm{H}^{i}\left(X, \wedge^{k} \mathfrak{n}\right)=\left\{\begin{array}{l}
0 \text { if } i \neq k, \\
\text { trivial } G \text {-module of dimension }|\{w \in W \mid l(w)=k\}| \text { if } i=k .
\end{array}\right.
$$

Finally, as part of the direct calculation one makes use of the following:

Lemma 3.1. If $\chi$ is a sum of distinct negative roots and $w \cdot \chi$ is dominant, then $w \cdot \chi=0$.

For a proof of this lemma see, for example, [J, 6.18 Proposition].

\section{Some Reductions}

Recall that we have reduced the question of normality and rational singularities to the study of

We have

$$
\mathrm{H}^{i}\left(\widetilde{\mathcal{A}}_{r}, \mathcal{O}_{\widetilde{\mathcal{A}}_{r}}\right)=\mathrm{H}^{i}\left(\left(T^{*} X\right)^{\oplus r}, \mathcal{O}_{\left(T^{*} X\right)^{\oplus r}}\right) .
$$

$$
\mathrm{H}^{i}\left(\widetilde{\mathcal{A}}_{r}, \mathcal{O}_{\widetilde{\mathcal{A}}_{r}}\right)=\mathrm{H}^{i}\left(\left(T^{*} X\right)^{\oplus r}, \mathcal{O}_{\left(T^{*} X\right)^{\oplus r}}\right)=\mathrm{H}^{i}\left(X, \operatorname{Sym}\left((\mathfrak{g} / \mathfrak{b})^{\oplus r}\right)\right) .
$$

In the latter equality we use the fact that the tangent bundle of $X$ is $T X=$ $\mathfrak{g} / \mathfrak{b}$ as $G$-equivariant vector bundles; recall our notational convention that the $B$ representation $\mathfrak{g} / \mathfrak{b}$ also stands for the corresponding $G$-equivariant bundle. Thus, we are reduced to analyzing the cohomology groups $\mathrm{H}^{i}\left(X, \operatorname{Sym}\left((\mathfrak{g} / \mathfrak{b})^{\oplus r}\right)\right)$. In particular, $\mathcal{A}_{r}$ is normal if and only if

$$
\mathbb{C}\left[\mathcal{A}_{r}\right]=\mathrm{H}^{0}\left(X, \operatorname{Sym}\left((\mathfrak{g} / \mathfrak{b})^{\oplus r}\right)\right) .
$$

We then conclude that

$\mathcal{A}_{r}$ is normal if and only if the map $\operatorname{Sym}\left(\mathfrak{g}^{\oplus r}\right) \rightarrow \mathrm{H}^{0}\left(X, \operatorname{Sym}\left((\mathfrak{g} / \mathfrak{b})^{\oplus r}\right)\right)$ is onto and

$$
\begin{aligned}
& \mathcal{A}_{r} \text { has rational singularities if and only if it is } \\
& \text { normal and } \mathrm{H}^{i}\left(X, \operatorname{Sym}\left((\mathfrak{g} / \mathfrak{b})^{\oplus r}\right)\right)=0 \text { for } i>0 .
\end{aligned}
$$

Next, we will make some very general reductions for the vanishing of the higher cohomology in (4.1). As we will show later, the higher cohomology does not vanish in general and hence the general reductions are not so useful. We will be able to obtain more precise statements later. However, the general remarks below are perhaps helpful as a general guide.

We begin with some simple lemmas:

Lemma 4.1. Suppose that $E$ is a $B$-module. Then for all $i \geq 1$,

$$
\mathrm{H}^{i}\left(X, \operatorname{Sym}\left(E^{\oplus r}\right)\right)=0 \text { for all } r \geq 1 \Longleftrightarrow \mathrm{H}^{i}\left(X, E^{\otimes r}\right)=0 \text { for all } r \geq 1 \text {. }
$$


Proof. We have $\operatorname{Sym}\left(E^{\oplus r}\right)=(\operatorname{Sym}(E))^{\otimes r}=\bigoplus\left(S^{i_{1}} E \otimes \cdots \otimes S^{i_{r}} E\right)$. So $E^{\otimes r}$ is a direct summand of $\operatorname{Sym}\left(E^{\oplus r}\right)$ and thus " $\Rightarrow$ " follows. In the other direction, assume that $\mathrm{H}^{i}\left(X, E^{\otimes r}\right)=0$ for all $r \geq 1$. Then we have $\mathrm{H}^{i}\left(X,\left(E^{\oplus r}\right)^{\otimes k}\right)=0$ for all $r, k \geq 1$. Now $\operatorname{Sym}^{k}\left(E^{\oplus r}\right)$ is a direct summand of $\left(E^{\oplus r}\right)^{\otimes k}$. It follows that $\mathrm{H}^{i}\left(X, \operatorname{Sym}^{k}\left(E^{\oplus r}\right)\right)=0$ for all $r, k \geq 1$ and thus $\mathrm{H}^{i}\left(X, \operatorname{Sym}\left(E^{\oplus r}\right)\right)=0$ for all $r \geq 1$.

In the same way we obtain

Lemma 4.2. Suppose that $E$ is a B-module. Then

$$
\begin{aligned}
& \mathrm{H}^{i}\left(X, \wedge^{r}\left(E^{\oplus k}\right)\right)=0 \text { for all } r, k \geq 1, i \geq r+1 \Longleftrightarrow \mathrm{H}^{i}\left(X, E^{\otimes r}\right)=0 \\
& \quad \text { for all } r \geq 1, i \geq r+1 .
\end{aligned}
$$

Let us now consider the vanishing statement:

$$
\mathrm{H}^{i}\left(X, \operatorname{Sym}\left((\mathfrak{g} / \mathfrak{b})^{\oplus r}\right)\right)=0 \text { for } i \geq 1 .
$$

Consider the short exact sequence

$$
0 \rightarrow \mathfrak{b}^{\oplus r} \rightarrow \mathfrak{g}^{\oplus r} \rightarrow(\mathfrak{g} / \mathfrak{b})^{\oplus r} \rightarrow 0 .
$$

Its associated Koszul complex provides a resolution of the $\operatorname{Sym}^{m}\left(\mathfrak{g}^{\oplus r}\right)$-module $\operatorname{Sym}^{m}\left((\mathfrak{g} / \mathfrak{b})^{\oplus r}\right)$ as follows:

$$
\cdots \wedge^{i}\left(\mathfrak{b}^{\oplus r}\right) \otimes \operatorname{Sym}^{m-i}\left(\mathfrak{g}^{\oplus r}\right) \rightarrow \cdots \rightarrow \operatorname{Sym}^{m}\left(\mathfrak{g}^{\oplus r}\right) \rightarrow \operatorname{Sym}^{m}\left((\mathfrak{g} / \mathfrak{b})^{\oplus r}\right) \rightarrow 0 .
$$

As the $\operatorname{Sym}^{m-i}\left(\mathfrak{g}^{\oplus r}\right)$ are $G$-representations, the corresponding $G$-equivariant vector bundles are trivial. We conclude that the vanishing statement (4.2) is equivalent to

$$
\mathrm{H}^{i+j}\left(X, \wedge^{j}\left(\mathfrak{b}^{\oplus r}\right)\right)=0 \text { for all } i \geq 1 .
$$

This statement holds for any particular $r$.

On the other hand, using Lemma 4.1, we see that the vanishing statement (4.2) for all $r$ is equivalent to

$$
\mathrm{H}^{i}\left(X,(\mathfrak{g} / \mathfrak{b})^{\otimes r}\right)=0 \text { for all } i, r \geq 1 .
$$

Similarly, using Lemma 4.2 we see that the vanishing statement (4.4) for all $r$ is equivalent to

$$
\mathrm{H}^{i}\left(X, \mathfrak{b}^{\otimes r}\right)=0 \text { for all } r \geq 1, i \geq r+1 .
$$

The short exact sequence

$$
0 \rightarrow \mathfrak{b} \rightarrow \mathfrak{g} \rightarrow \mathfrak{g} / \mathfrak{b} \rightarrow 0
$$

will give us further information. If we consider it as a two-step complex $\mathfrak{b} \rightarrow \mathfrak{g}$ with cohomology $\mathfrak{g} / \mathfrak{b}$ and pass to the associated tensor complexes, we obtain

$$
\cdots\left(\mathfrak{g}^{\otimes r-q} \otimes \mathfrak{b}^{\otimes q}\right)^{\oplus\left(\begin{array}{c}
r \\
q
\end{array}\right)} \rightarrow \cdots \rightarrow \mathfrak{g}^{\otimes r} \rightarrow(\mathfrak{g} / \mathfrak{b})^{\otimes r} \rightarrow 0 .
$$

Passing to the associated spectral sequence we obtain

$$
E_{1}^{p,-q}=\left(\mathfrak{g}^{\otimes r-q}\right)^{\oplus\left(\begin{array}{l}
r \\
q
\end{array}\right)} \otimes \mathrm{H}^{p}\left(X, \mathfrak{b}^{\otimes q}\right) \quad \Longrightarrow \mathrm{H}^{p-q}\left(X,(\mathfrak{g} / \mathfrak{b})^{\otimes r}\right) .
$$

From this spectral sequence we conclude that the condition

$$
\mathrm{H}^{i}\left(X, \mathfrak{b}^{\otimes r}\right)=0 \text { for all } i \geq r
$$

implies

$$
\mathfrak{g}^{\otimes r} \rightarrow \mathrm{H}^{0}\left(X,(\mathfrak{g} / \mathfrak{b})^{\otimes r}\right) \quad \text { is a surjection . }
$$


Arguing as in Lemma 4.1 by decomposing tensors we conclude that if condition (4.6) holds for all $r$, then $\operatorname{Sym}\left(\mathfrak{g}^{\oplus r}\right) \rightarrow \mathrm{H}^{0}\left(X, \operatorname{Sym}\left((\mathfrak{g} / \mathfrak{b})^{\oplus r}\right)\right)$ is onto for all $r$. Combining this with the previous discussion we obtain

Theorem 4.3. If condition (4.6) is satisfied for all $r$, then $\mathcal{A}_{r}$ has rational singularities for all values $r$.

\section{Cohomology of the Vector Bundles $\mathfrak{b}^{\otimes r}$ FOR Type $A_{n}$}

In the previous section we reduced the question of normality and the question of rational singularities of $\mathcal{A}_{r}$ to the study of the cohomology of $\mathfrak{b}^{\otimes r}$. We will now calculate the cohomology of these vector bundles for small $r$ in type $A_{n-1}$, i.e., for $\mathfrak{g}=\mathfrak{s l}_{n}$. We will go sufficiently far to obtain our counterexamples, but will not make an attempt for a complete general answer.

5.1. The case $r=1$ and $\mathfrak{g}$ of any type. This case is, of course, well known, easy and applies to any $\mathfrak{g}$, but we include the details in any case.

We will show that:

$$
\mathrm{H}^{k}(X, \mathfrak{b})=0 \text { for all } k .
$$

Consider the short exact sequence

$$
0 \rightarrow \mathfrak{n} \rightarrow \mathfrak{b} \rightarrow \mathfrak{b} / \mathfrak{n} \rightarrow 0
$$

Since $\mathfrak{b} / \mathfrak{n}$ is a trivial vector bundle, $\mathrm{H}^{k}(X, \mathfrak{b} / \mathfrak{n})=0$ for all $k \geq 1$. As by (3.2) we have $\mathrm{H}^{k}(X, \mathfrak{n})=0$ for $k \neq 1$, it follows that

$$
\mathrm{H}^{k}(X, \mathfrak{b})=0 \text { for } k \geq 2 .
$$

From the short exact sequence

$$
0 \rightarrow \mathfrak{b} \rightarrow \mathfrak{g} \rightarrow \mathfrak{g} / \mathfrak{b} \rightarrow 0
$$

we obtain the exact sequence

$$
0 \rightarrow \mathrm{H}^{0}(X, \mathfrak{b}) \rightarrow \mathrm{H}^{0}(X, \mathfrak{g}) \rightarrow \mathrm{H}^{0}(X, \mathfrak{g} / \mathfrak{b}) \rightarrow \mathrm{H}^{1}(X, \mathfrak{b}) \rightarrow 0 .
$$

Moreover, $\mathrm{H}^{0}(X, \mathfrak{g}) \cong \mathfrak{g}$ and the map $\mathfrak{g} \rightarrow \mathrm{H}^{0}(X, \mathfrak{g} / \mathfrak{b})$ is an isomorphism, as can be seen by a direct verification, for example. Thus, we obtain (5.1).

5.2. The case $r=2$ and $\mathfrak{g}$ of type $A_{n-1}$. Let $\mathfrak{g}=\mathfrak{s l}_{n}, n \geq 3$. We will show that

$$
\mathrm{H}^{k}(X, \mathfrak{b} \otimes \mathfrak{b})= \begin{cases}0 & \text { if } k \neq 1 \\ \mathbb{C} & \text { if } k=1\end{cases}
$$

and

$$
\mathrm{H}^{k}\left(X,(\mathfrak{g} / \mathfrak{b})^{\otimes 2}\right) \cong \begin{cases}(\mathfrak{g} \otimes \mathfrak{g}) / \mathbb{C} & \text { if } k=0 \\ 0 & \text { if } k \geq 1\end{cases}
$$

First, we claim that

$$
\text { the potential support of } \mathrm{H}^{k}(X, \mathfrak{b} \otimes \mathfrak{b}) \text { is }\{0\} \text { for all } k \geq 0 \text {. }
$$

Let $\chi \in \chi(\mathfrak{b} \otimes \mathfrak{b})$. We will show that

$$
\text { either } \mathrm{H}^{k}\left(X, \mathcal{L}_{\chi}\right)=0 \text { for all } k \geq 0 \text { or } \chi=w \cdot 0 \text { for some } w \in W \text {. }
$$

Recall that the bundle associated to $\mathfrak{h}=\mathfrak{b} / \mathfrak{n}$ is trivial and thus, by making use of Lemma 3.1, it suffices to prove the result for $\chi \in \chi(\mathfrak{n} \otimes \mathfrak{n})$. The case when $\chi$ is a sum of distinct negative roots follows from (3.1) and Lemma 3.1. So it remains to 
consider the case when $\chi \in \chi(\mathfrak{n} \otimes \mathfrak{n})$ and $\chi$ is not a sum of distinct negative roots. In that case $\chi=-2 \alpha$ for a positive root $\alpha \in \Phi^{+}$and $\operatorname{ht}(\alpha)=1$ (if $\operatorname{ht}(\alpha)>1$, then $\chi$ is a sum of distinct negative roots). Let us denote the set of simple roots with respect to $\Phi^{+}$by $\Delta:=\left\{\alpha_{i}, i=1, \ldots, n-1\right\}$. Let $\alpha=\alpha_{i}, 1 \leq i \leq n-1$. If $i \leq n-2$, then $\left\langle-2 \alpha_{i}+\rho,\left(\alpha_{i}+\alpha_{i+1}\right)^{\vee}\right\rangle=0$; if $i \geq 2$, then $\left\langle-2 \alpha_{i}+\rho,\left(\alpha_{i-1}+\alpha_{i}\right)^{\vee}\right\rangle=0$; thus, $\mathrm{H}^{k}\left(X, \mathcal{L}_{\chi}\right)=0$ for all $k$. This finishes the proof of (5.4).

Using the short exact sequence

$$
0 \rightarrow \mathfrak{b} \otimes \mathfrak{b} \rightarrow \mathfrak{g} \otimes \mathfrak{b} \rightarrow \mathfrak{g} / \mathfrak{b} \otimes \mathfrak{b} \rightarrow 0
$$

and $\mathrm{H}^{k}(X, \mathfrak{g} \otimes \mathfrak{b}) \cong \mathfrak{g} \otimes \mathrm{H}^{k}(X, \mathfrak{b})=0$ for all $k$ (we make use of (5.1) $)$, we see that $\mathrm{H}^{0}(X, \mathfrak{b} \otimes \mathfrak{b})=0$ and $\mathrm{H}^{k}(X, \mathfrak{b} \otimes \mathfrak{b}) \cong \mathrm{H}^{k-1}(X, \mathfrak{b} \otimes \mathfrak{g} / \mathfrak{b})$ as $G$-modules for $k \geq 1$.

Using the short exact sequence

$$
0 \rightarrow \mathfrak{b} \otimes \mathfrak{g} / \mathfrak{b} \rightarrow \mathfrak{g} \otimes \mathfrak{g} / \mathfrak{b} \rightarrow(\mathfrak{g} / \mathfrak{b})^{\otimes 2} \rightarrow 0
$$

and $H^{k}(X, \mathfrak{g} \otimes \mathfrak{g} / \mathfrak{b}) \cong \mathfrak{g} \otimes \mathrm{H}^{k}(X, \mathfrak{g} / \mathfrak{b})=0$ for $k \geq 1$, we see that

$$
\mathrm{H}^{k}(X, \mathfrak{b} \otimes \mathfrak{g} / \mathfrak{b}) \cong \mathrm{H}^{k-1}\left(X,(\mathfrak{g} / \mathfrak{b})^{\otimes 2}\right) \text { as } G \text {-modules for } k \geq 2
$$

and that we have an exact sequence of $G$-modules

$$
0 \rightarrow \mathrm{H}^{0}(X, \mathfrak{b} \otimes \mathfrak{g} / \mathfrak{b}) \rightarrow \mathfrak{g} \otimes \mathfrak{g} \rightarrow \mathrm{H}^{0}\left(X,(\mathfrak{g} / \mathfrak{b})^{\otimes 2}\right) \rightarrow \mathrm{H}^{1}(X, \mathfrak{b} \otimes \mathfrak{g} / \mathfrak{b}) \rightarrow 0 .
$$

It follows that we have an isomorphism of $G$-modules

$$
\mathrm{H}^{k}(X, \mathfrak{b} \otimes \mathfrak{b}) \cong \mathrm{H}^{k-2}\left(X,(\mathfrak{g} / \mathfrak{b})^{\otimes 2}\right) \text { for } k \geq 3
$$

and an exact sequence of $G$-modules

$$
0 \rightarrow \mathrm{H}^{1}(X, \mathfrak{b} \otimes \mathfrak{b}) \rightarrow \mathfrak{g} \otimes \mathfrak{g} \rightarrow \mathrm{H}^{0}\left(X,(\mathfrak{g} / \mathfrak{b})^{\otimes 2}\right) \rightarrow \mathrm{H}^{2}(X, \mathfrak{b} \otimes \mathfrak{b}) \rightarrow 0 .
$$

As 0 is clearly not in the potential support of $\mathrm{H}^{k}\left(X,(\mathfrak{g} / \mathfrak{b})^{\otimes 2}\right)$, it follows from (5.4), (5.5) and (5.6) that $\mathrm{H}^{k}(X, \mathfrak{b} \otimes \mathfrak{b})=0$ for $k \geq 2$, and

$$
\mathrm{H}^{1}(X, \mathfrak{b} \otimes \mathfrak{b}) \cong \operatorname{Hom}_{G}(\mathbb{C}, \mathfrak{g} \otimes \mathfrak{g}) \cong \operatorname{Hom}_{G}(\mathfrak{g}, \mathfrak{g}) \cong \mathbb{C}
$$

This completes the proof of (5.2). It also follows that

$$
\mathrm{H}^{k}(X, \mathfrak{b} \otimes \mathfrak{g} / \mathfrak{b}) \cong \begin{cases}\mathbb{C} & \text { if } k=0 \\ 0 & \text { if } k \geq 1\end{cases}
$$

Finally, we conclude (5.3) from (5.2), (5.5), and (5.6).

5.3. The case $r=3$ and $\mathfrak{g}$ of type $A_{n-1}$. Let $\mathfrak{g}=\mathfrak{s l}_{n}, n \geq 3$. We will show that

$$
\begin{aligned}
\mathrm{H}^{k}\left(X, \mathfrak{b}^{\otimes 3}\right) & =0, k \leq 1 \text { or } k \geq 4, \\
\mathrm{H}^{2}\left(X, \mathfrak{b}^{\otimes 3}\right) & = \begin{cases}\mathbb{C}^{\oplus 2} \oplus L\left(\alpha_{1}+\alpha_{2}\right)^{\oplus 5} \oplus L\left(2 \alpha_{1}+\alpha_{2}\right) & \text { if } n=3, \\
\mathbb{C}^{\oplus 2} & \text { if } n \geq 4,\end{cases} \\
\mathrm{H}^{3}\left(X, \mathfrak{b}_{1} \otimes 3\right. & = \begin{cases}L\left(\alpha_{1}+2 \alpha_{2}+\alpha_{3}\right) & \text { if } n=4, \\
0 & \text { if } n \neq 4 .\end{cases}
\end{aligned}
$$


We first show that

$$
\begin{aligned}
& \text { if } n \geq 5 \quad \operatorname{PSupp}\left(\mathrm{H}^{k}\left(X, \mathfrak{b}^{\otimes 3}\right)\right)=\{0\} \text { for all } k, \\
& \text { if } n=4 \quad \operatorname{PSupp}\left(\mathrm{H}^{k}\left(X, \mathfrak{b}^{\otimes 3}\right)\right)= \begin{cases}\left\{0, \alpha_{1}+2 \alpha_{2}+\alpha_{3}\right\} & \text { if } k=3, \\
\{0\} & \text { if } k \neq 3,\end{cases} \\
& \text { if } n=3 \quad \operatorname{PSupp}\left(\mathrm{H}^{k}\left(X, \mathfrak{b}^{\otimes 3}\right)\right) \\
& = \begin{cases}\left\{0, \alpha_{1}+\alpha_{2}, 2 \alpha_{1}+\alpha_{2}, \alpha_{1}+2 \alpha_{2}\right\} & \text { if } k=2, \\
\left\{0, \alpha_{1}+\alpha_{2}\right\} & \text { if } k=3, \\
\{0\} & \text { if } k \neq 2,3 .\end{cases}
\end{aligned}
$$

Let $\chi \in \chi\left(\mathfrak{b}^{\otimes 3}\right)$. Recall that the bundle associated to $\mathfrak{h}=\mathfrak{b} / \mathfrak{n}$ is trivial and thus, by making use of (5.4), it suffices to prove the result for $\chi \in \chi\left(\mathfrak{n}^{\otimes 3}\right)$. If $\chi \in \chi\left(\wedge^{3} \mathfrak{n}\right)$, we see by (3.1) and Lemma 3.1 that either $H^{k}\left(X, \mathcal{L}_{\chi}\right)=0$ for all $k$ or $\chi=w \cdot 0$ for some $w \in W$. It remains to consider $\chi$ of the form $-2 \alpha-\beta, \alpha, \beta \in \Phi^{+}$.

Let us introduce some notation. For $w \in W$, denote

$$
\Phi_{w}:=\left\{\gamma \in \Phi^{+} \mid w^{-1} \gamma \in \Phi^{-}\right\} .
$$

Suppose that $w=s_{i_{1}} s_{i_{2}} \cdots s_{i_{l}}$, where $s_{i}=s_{\alpha_{i}}$, is a reduced expression. Recall that we have

$$
\Phi_{w}=\left\{\alpha_{i_{1}}, s_{i_{1}}\left(\alpha_{i_{2}}\right), s_{i_{1}} s_{i_{2}}\left(\alpha_{i_{3}}\right), \cdots, s_{i_{1}} \cdots s_{i_{l-1}}\left(\alpha_{i_{l}}\right)\right\} .
$$

In particular, the cardinality of $\Phi_{w}$ is the length $\ell(w)$.

For $\lambda \in X(T)$, we have

$$
w \cdot \lambda=w(\lambda)-\sum_{\gamma \in \Phi_{w}} \gamma
$$

Suppose first that $\alpha \neq \beta$. We show that if there exists $w \in W$ such that $w \cdot(-2 \alpha-\beta) \in X^{+}(T)$, then $w \cdot(-2 \alpha-\beta)=0$ unless $n=3$ and $\chi=-3 \alpha_{1}-\alpha_{2}$ or $-\alpha_{1}-3 \alpha_{2}$.

As the dominant Weyl chamber is contained in the positive root cone, i.e., the inverse of the Cartan matrix has positive entries, we have:

Let $\lambda \in \mathbb{Z} \Phi$ be an element in the root lattice which is dominant and not zero, then $\lambda \in \mathbb{Z}_{>0} \alpha_{1}+\cdots+\mathbb{Z}_{>0} \alpha_{n-1}$.

Assume that $w \cdot(-2 \alpha-\beta)$ is dominant and not equal to zero. Making use of (5.10) and (5.11) we see that

$$
w \cdot(-2 \alpha-\beta)=-2 w \alpha-w \beta-\sum_{\gamma \in \Phi_{w}} \gamma \in \mathbb{Z}_{>0} \alpha_{1}+\cdots+\mathbb{Z}_{>0} \alpha_{n-1} .
$$

Let us write $\alpha_{0}=\alpha_{1}+\cdots+\alpha_{n-1}$ for the highest root and then we can rephrase the above equality as

$$
-2 w \alpha-w \beta-\sum_{\gamma \in \Phi_{w}} \gamma \geq \alpha_{0} .
$$

Clearly, at least one of $w \alpha$ or $w \beta$ has to be negative. Note that if $w \beta$ is negative, then $-w \beta \in \Phi_{w}$ and similarly for $w \alpha$. Thus, if $w \alpha$ is negative and $w \beta$ is not, we get

$$
-w \alpha-w \beta-\sum_{\gamma \in \Phi_{w}-\{-w \alpha\}} \gamma \geq \alpha_{0} .
$$


But this is impossible as $-w \alpha \leq \alpha_{0}$. Similarly, we see that it is impossible for $w \alpha$ to be positive and for $w \beta$ to be negative. Hence, both $w \alpha$ and $w \beta$ have to be negative. In this case we see that

$$
-w \alpha-\sum_{\gamma \in \Phi_{w}-\{-w \alpha,-w \beta\}} \gamma \geq \alpha_{0} .
$$

This is only possble if $w \alpha=-\alpha_{0}, \Phi_{w}=\left\{\alpha_{0},-w \beta\right\}$, and then also $\ell(w)=2$. One sees directly that $w \alpha_{0}$ can be negative for $\ell(w)=2$ only when $n=3$. In that case the only possibilities are:

$$
-2 \alpha_{1}-\left(\alpha_{1}+\alpha_{2}\right)=\left(s_{1} s_{2}\right) \cdot\left(\alpha_{1}+\alpha_{2}\right), \quad-2 \alpha_{2}-\left(\alpha_{1}+\alpha_{2}\right)=\left(s_{2} s_{1}\right) \cdot\left(\alpha_{1}+\alpha_{2}\right) .
$$

Thus, we conclude that in this case the only possibility for potential support, in addition to 0 , is $\alpha_{1}+\alpha_{2} \in \operatorname{PSuppH}^{2}\left(X, \mathfrak{b}^{\otimes 3}\right)$ when $n=3$.

Suppose now that $\alpha=\beta$ and thus $\chi=-3 \alpha$. If ht $\alpha \geq 3$, then $\chi$ is a sum of distinct negative roots and we see by (3.1) and Lemma 3.1 that either $H^{k}\left(X, \mathcal{L}_{\chi}\right)=$ 0 for all $k$ or $\chi=w \cdot 0$ for some $w \in W$. Let us assume next that ht $\alpha=2$ and we write $\alpha=\alpha_{i}+\alpha_{i+1}, 1 \leq i \leq n-2$. If $i \leq n-3$, then $\left\langle-3 \alpha+\rho,\left(\alpha_{i}+\alpha_{i+1}+\alpha_{i+2}\right)^{\vee}\right\rangle=$ 0 and if $i \geq 2$, then $\left\langle-3 \alpha+\rho,\left(\alpha_{i-1}+\alpha_{i}+\alpha_{i+1}\right)^{\vee}\right\rangle=0$. Thus, in these cases $\mathrm{H}^{k}\left(X, \mathcal{L}_{\chi}\right)=0$ for all $k$ and they do not contribute to the potential support. Therefore, we are left to consider the case when $n=3$ and $i=1$. Then

$$
-3\left(\alpha_{1}+\alpha_{2}\right)=\left(s_{1} s_{2} s_{1}\right) \cdot\left(\alpha_{1}+\alpha_{2}\right) .
$$

Hence, $\alpha_{1}+\alpha_{2} \in \operatorname{PSuppH}^{3}\left(X, \mathfrak{b}^{\otimes 3}\right)$ when $n=3$.

Finally, let us assume that ht $\alpha=1$ and so $\alpha=\alpha_{i}, 1 \leq i \leq n-1$. If $i \leq n-3$, we have $\left\langle-3 \alpha+\rho,\left(\alpha_{i}+\alpha_{i+1}+\alpha_{i+2}\right)^{\vee}\right\rangle=0$; if $i \geq 3$, we have $\left\langle-3 \alpha+\rho,\left(\alpha_{i-2}+\alpha_{i-1}+\right.\right.$ $\left.\left.\alpha_{i}\right)^{\vee}\right\rangle=0$. This shows that $\mathrm{H}^{k}\left(X, \mathcal{L}_{\chi}\right)=0$ for all $k$. Thus, we get no contribution to the potential support in these cases. This leaves us with the possibilities of $n=3$ with $i=1,2$ and $n=4$ with $i=2$. In these cases, we have

$$
\begin{gathered}
-3 \alpha_{1}=\left(s_{1} s_{2}\right) \cdot\left(2 \alpha_{1}+\alpha_{2}\right), \quad-3 \alpha_{2}=\left(s_{2} s_{1}\right) \cdot\left(\alpha_{1}+2 \alpha_{2}\right) \quad(n=3) \\
-3 \alpha_{2}=\left(s_{2} s_{1} s_{3}\right) \cdot\left(\alpha_{1}+2 \alpha_{2}+\alpha_{3}\right) \quad(n=4) .
\end{gathered}
$$

Thus, we obtain $2 \alpha_{1}+\alpha_{2}, \alpha_{1}+2 \alpha_{2} \in \operatorname{PSuppH}^{2}\left(X, \mathfrak{b}^{\otimes 3}\right)$ when $n=3$ and $\alpha_{1}+2 \alpha_{2}+$ $\alpha_{3} \in \operatorname{PSuppH}^{3}\left(X, \mathfrak{b}^{\otimes 3}\right)$ when $n=4$.

This completes the proof of (5.9) and we now turn to the proof of (5.8).

Using the short exact sequence

$$
0 \rightarrow \mathfrak{b}^{\otimes 3} \rightarrow \mathfrak{g} \otimes \mathfrak{b}^{\otimes 2} \rightarrow \mathfrak{b}^{\otimes 2} \otimes \mathfrak{g} / \mathfrak{b} \rightarrow 0
$$

and (5.2) we see that we have

$$
\mathrm{H}^{0}\left(X, \mathfrak{b}^{\otimes 3}\right)=0, \mathrm{H}^{k}\left(X, \mathfrak{b}^{\otimes 3}\right) \cong \mathrm{H}^{k-1}\left(X, \mathfrak{b}^{\otimes 2} \otimes \mathfrak{g} / \mathfrak{b}\right) \text { for } k \geq 3,
$$

and also an exact sequence

$0 \rightarrow \mathrm{H}^{0}\left(X, \mathfrak{b}^{\otimes 2} \otimes \mathfrak{g} / \mathfrak{b}\right) \rightarrow \mathrm{H}^{1}\left(X, \mathfrak{b}^{\otimes 3}\right) \rightarrow \mathfrak{g} \rightarrow \mathrm{H}^{1}\left(X, \mathfrak{b}^{\otimes 2} \otimes \mathfrak{g} / \mathfrak{b}\right) \rightarrow \mathrm{H}^{2}\left(X, \mathfrak{b}^{\otimes 3}\right) \rightarrow 0$.

Using the short exact sequence

$$
0 \rightarrow \mathfrak{b}^{\otimes 2} \otimes \mathfrak{g} / \mathfrak{b} \rightarrow \mathfrak{g} \otimes \mathfrak{b} \otimes \mathfrak{g} / \mathfrak{b} \rightarrow \mathfrak{b} \otimes(\mathfrak{g} / \mathfrak{b})^{\otimes 2} \rightarrow 0
$$

and (5.7) we obtain

$$
\mathrm{H}^{k}\left(X, \mathfrak{b}^{\otimes 2} \otimes \mathfrak{g} / \mathfrak{b}\right) \cong \mathrm{H}^{k-1}\left(X, \mathfrak{b} \otimes(\mathfrak{g} / \mathfrak{b})^{\otimes 2}\right) \text { for } k \geq 2
$$


and an exact sequence

(5.13) $0 \rightarrow \mathrm{H}^{0}\left(X, \mathfrak{b}^{\otimes 2} \otimes \mathfrak{g} / \mathfrak{b}\right) \rightarrow \mathfrak{g} \rightarrow \mathrm{H}^{0}\left(X, \mathfrak{b} \otimes(\mathfrak{g} / \mathfrak{b})^{\otimes 2}\right) \rightarrow \mathrm{H}^{1}\left(X, \mathfrak{b}^{\otimes 2} \otimes \mathfrak{g} / \mathfrak{b}\right) \rightarrow 0$.

Using the short exact sequence

$$
0 \rightarrow \mathfrak{b} \otimes(\mathfrak{g} / \mathfrak{b})^{\otimes 2} \rightarrow \mathfrak{g} \otimes(\mathfrak{g} / \mathfrak{b})^{\otimes 2} \rightarrow(\mathfrak{g} / \mathfrak{b})^{\otimes 3} \rightarrow 0
$$

and (5.3) we obtain

$$
\mathrm{H}^{k}\left(X, \mathfrak{b} \otimes(\mathfrak{g} / \mathfrak{b})^{\otimes 2}\right) \cong \mathrm{H}^{k-1}\left(X,(\mathfrak{g} / \mathfrak{b})^{\otimes 3}\right) \text { for } k \geq 2
$$

and an exact sequence

$$
\begin{aligned}
0 \rightarrow \mathrm{H}^{0}\left(X, \mathfrak{b} \otimes(\mathfrak{g} / \mathfrak{b})^{\otimes 2}\right) & \rightarrow \mathfrak{g} \otimes \mathrm{H}^{0}\left(X,(\mathfrak{g} / \mathfrak{b})^{\otimes 2}\right) \rightarrow \mathrm{H}^{0}\left(X, \mathfrak{g} / \mathfrak{b}^{\otimes 3}\right) \\
& \rightarrow \mathrm{H}^{1}\left(X, \mathfrak{b} \otimes(\mathfrak{g} / \mathfrak{b})^{\otimes 2}\right) \rightarrow 0 .
\end{aligned}
$$

It follows that we have an isomorphism of $G$-modules

$$
\mathrm{H}^{k}\left(X, \mathfrak{b}^{\otimes 3}\right) \cong \mathrm{H}^{k-3}\left(X,(\mathfrak{g} / \mathfrak{b})^{\otimes 3}\right) \text { for } k \geq 4,
$$

and an exact sequence of $G$-modules

$$
\begin{aligned}
0 \rightarrow \mathrm{H}^{0}\left(X, \mathfrak{b} \otimes(\mathfrak{g} / \mathfrak{b})^{\otimes 2}\right) & \rightarrow \mathfrak{g} \otimes \mathrm{H}^{0}\left(X,(\mathfrak{g} / \mathfrak{b})^{\otimes 2}\right) \\
& \rightarrow \mathrm{H}^{0}\left(X,(\mathfrak{g} / \mathfrak{b})^{\otimes 3}\right) \rightarrow \mathrm{H}^{3}\left(X, \mathfrak{b}^{\otimes 3}\right) \rightarrow 0 .
\end{aligned}
$$

Thus, as 0 is not in the potential support of $\mathrm{H}^{k}\left(X,(\mathfrak{g} / \mathfrak{b})^{\otimes 3}\right)$, we conclude that

$$
\mathrm{H}^{k}\left(X, \mathfrak{b}^{\otimes 3}\right) \quad \text { does not contain the trivial representation for } k \geq 3 .
$$

Thus, we conclude from (5.9) that $\mathrm{H}^{k}\left(X, \mathfrak{b}^{\otimes 3}\right)=0$ for $k \geq 4$.

Also, it follows from (5.9), (5.12), and (5.13) that

$$
\mathrm{H}^{0}\left(X, \mathfrak{b}^{\otimes 2} \otimes \mathfrak{g} / \mathfrak{b}\right) \cong \mathrm{H}^{1}\left(X, \mathfrak{b}^{\otimes 3}\right)=0 .
$$

Thus, we have shown, in particular, that $\mathrm{H}^{k}\left(X, \mathfrak{b}^{\otimes 3}\right)=0$ for $k=0,1$. Thus, we have obtained the first claim of (5.8). Before proceeding further, we record one more general fact which we obtain from (5.16), (5.12), and (5.13):

$$
\begin{gathered}
\mathrm{H}^{0}\left(X, \mathfrak{b} \otimes(\mathfrak{g} / \mathfrak{b})^{\otimes 2}\right) \cong \mathrm{H}^{1}\left(X, \mathfrak{b}^{\otimes 2} \otimes \mathfrak{g} / \mathfrak{b}\right) \oplus \mathfrak{g} \cong \mathrm{H}^{2}\left(X, \mathfrak{b}^{\otimes 3}\right) \oplus \mathfrak{g}^{\oplus 2}, \\
\mathrm{H}^{1}\left(X, \mathfrak{b} \otimes(\mathfrak{g} / \mathfrak{b})^{\otimes 2}\right) \cong \mathrm{H}^{2}\left(X, \mathfrak{b}^{\otimes 2} \otimes \mathfrak{g} / \mathfrak{b}\right) \cong \mathrm{H}^{3}\left(X, \mathfrak{b}^{\otimes 3}\right), \\
\mathrm{H}^{k-1}\left(X, \mathfrak{b} \otimes(\mathfrak{g} / \mathfrak{b})^{\otimes 2}\right) \cong \mathrm{H}^{k}\left(X, \mathfrak{b}^{\otimes 2} \otimes \mathfrak{g} / \mathfrak{b}\right)=0 \text { if } k \neq 1,2 .
\end{gathered}
$$

We now argue with specific values of $n$.

Assume that $n \geq 5$. It follows from (5.15) and (5.9) that

$$
\mathrm{H}^{3}\left(X, \mathfrak{b}^{\otimes 3}\right)=0 \text { and } \mathrm{H}^{2}\left(X, \mathfrak{b}^{\otimes 3}\right) \cong \mathbb{C}^{\oplus c},
$$

for some $c$ which we determine later.

Assume that $n=4$. It follows from (5.15) and (5.9) that

$$
\mathrm{H}^{3}\left(X, \mathfrak{b}^{\otimes 3}\right) \cong L\left(\alpha_{1}+2 \alpha_{2}+\alpha_{3}\right) \text { and } \mathrm{H}^{2}\left(X, \mathfrak{b}^{\otimes 3}\right) \cong \mathbb{C}^{\oplus a},
$$

for some $a$ which we determine later.

Assume that $n=3$. As $\alpha_{1}+\alpha_{2}$ is not in the potential support of $\mathrm{H}^{0}\left(X,(\mathfrak{g} / \mathfrak{b})^{\otimes 3}\right)$, we conclude from (5.9), (5.14) and (5.15) that

$$
\mathrm{H}^{3}\left(X, \mathfrak{b}^{\otimes 3}\right)=0 .
$$


Thus, $\mathrm{H}^{2}\left(X, \mathfrak{b}^{\otimes 3}\right)$ is the only non-vanishing cohomology group in this case. Making use of Borel-Weil-Bott (3.1), just as in our argument for (5.9) we see that

$$
\mathrm{H}^{*}\left(X, \operatorname{gr}\left(\mathfrak{b}^{\otimes 3}\right)\right) \cong \mathbb{C}^{\oplus b}+L\left(\alpha_{1}+\alpha_{2}\right)^{\oplus 5}+L\left(2 \alpha_{1}+\alpha_{2}\right)+L\left(\alpha_{1}+2 \alpha_{2}\right) .
$$

for some $b$. Therefore, we get:

$\mathrm{H}^{2}\left(X, \mathfrak{b}^{\otimes 3}\right) \cong \mathrm{H}^{*}\left(X, \operatorname{gr}\left(\mathfrak{b}^{\otimes 3}\right)\right) \cong \mathbb{C}^{\oplus b} \oplus L\left(\alpha_{1}+\alpha_{2}\right)^{\oplus 5} \oplus L\left(2 \alpha_{1}+\alpha_{2}\right) \oplus L\left(\alpha_{1}+2 \alpha_{2}\right)$.

We now determine $a, b$, and $c$. Now,

$$
\mathbb{C}^{\oplus a} \cong \mathbb{C}^{\oplus b} \cong \mathbb{C}^{\oplus c} \cong \operatorname{Hom}_{G}\left(\mathbb{C}, \mathrm{H}^{2}\left(X, \mathfrak{b}^{\otimes 3}\right)\right) .
$$

By (5.17a) we get

$$
\begin{aligned}
\operatorname{Hom}_{G}\left(\mathbb{C}, H^{2}\left(X, \mathfrak{b}^{\otimes 3}\right)\right) & \cong \operatorname{Hom}_{G}\left(\mathbb{C}, \mathrm{H}^{2}\left(X, \mathfrak{b}^{\otimes 3}\right) \oplus \mathfrak{g}^{\oplus 2}\right) \\
& \cong \operatorname{Hom}_{G}\left(\mathbb{C}, \mathrm{H}^{0}\left(X, \mathfrak{b} \otimes(\mathfrak{g} / \mathfrak{b})^{\otimes 2}\right)\right) \\
& \cong \operatorname{Hom}_{G}\left(\mathbb{C}, \mathfrak{g} \otimes \mathrm{H}^{0}\left(X,(\mathfrak{g} / \mathfrak{b})^{\otimes 2}\right)\right) \cong(\mathfrak{g} \otimes \mathfrak{g} \otimes \mathfrak{g})^{G} \cong \mathbb{C}^{\oplus 2}
\end{aligned}
$$

where in the third equality we have made use of (5.14) and the fact that the trivial representation does not occur in $\mathrm{H}^{0}\left(X,(\mathfrak{g} / \mathfrak{b})^{\otimes 3}\right)$; in the fourth equality we made use of (5.3). The last equality is classical and can also be verified by a direct calculation: the two invariant tensors are $(x, y, z) \mapsto \operatorname{Tr}(x y z)$ and $(x, y, z) \mapsto \operatorname{Tr}(y x z)$. This completes the proof of (5.8).

5.4. The case $r=4$ and $\mathfrak{g}$ of type $A_{n-1}$. Let $\mathfrak{g}=\mathfrak{s l}_{n}, n \geq 6$.

In the previous cases we obtained complete information of the cohomology for all values of $n \geq 3$. For $r=4$ we will not make an attempt to get a complete answer, but will just obtain enough information for our counterexample. In particular, we already have enough information to prove the Cohen-Macaulay property for $\mathfrak{s l}_{3}$. In the cases $n=4,5$ the answer is probably obtainable with our techniques, but is more complicated.

We will show that if $n \geq 7$, then

$$
\mathrm{H}^{k}\left(X, \mathfrak{b}^{\otimes 4}\right)=0 \text { if } k \neq 2,3
$$

and if $n=6$, then

$$
\mathrm{H}^{k}\left(X, \mathfrak{b}^{\otimes 4}\right)= \begin{cases}L\left(\alpha_{1}+2 \alpha_{2}+3 \alpha_{3}+2 \alpha_{4}+\alpha_{5}\right) & \text { if } k=5 \\ 0 & \text { if } k \neq 2,3,5 .\end{cases}
$$

Remark 5.1. The $\mathrm{H}^{2}\left(X, \mathfrak{b}^{\otimes 4}\right)$ and $\mathrm{H}^{3}\left(X, \mathfrak{b}^{\otimes 4}\right)$ both consist of a number of copies of the trivial representation.

We first show that

$$
\text { if } n \geq 7 \quad \operatorname{PSuppH}^{k}\left(X, \mathfrak{b}^{\otimes 4}\right)=\{0\} \text { for all } k \geq 0
$$

and

$$
\text { if } \begin{aligned}
n & =6 \operatorname{PSuppH}^{k}\left(X, \mathfrak{b}^{\otimes 4}\right) \\
& = \begin{cases}\{0\} & \text { if } k \neq 5, \\
\left\{0, \alpha_{1}+2 \alpha_{2}+3 \alpha_{3}+2 \alpha_{4}+\alpha_{5}\right\} & \text { if } k=5 .\end{cases}
\end{aligned}
$$

Let $\chi \in \chi\left(\mathfrak{b}^{\otimes 4}\right)$. Recall that the bundle associated to $\mathfrak{h}=\mathfrak{b} / \mathfrak{n}$ is trivial. Thus, by making use of (5.9), it suffices to prove the result for $\chi \in \chi\left(\mathfrak{n}^{\otimes 4}\right)$. If $\chi \in \chi\left(\wedge^{4} \mathfrak{n}\right)$, we see by (3.1) and Lemma 3.1 that either $H^{k}\left(X, \mathcal{L}_{\chi}\right)=0$ for all $k$ or $\chi=w \cdot 0$ for 
some $w \in W$. Thus, we are reduced to considering the case when $\chi$ is not a sum of distinct roots.

Assume first that $\chi=-2 \alpha-\beta-\gamma$, where $\alpha, \beta, \gamma \in \Phi^{+}$are distinct. We will make use of the notation and argue in a similar manner as in subsection 5.3. Suppose that there exists a $w \in W$ such that $w \cdot \chi \in X^{+}(T)$ and $w \cdot \chi \neq 0$. Making use of (5.10) and (5.11) we conclude, as in subsection 5.3, that

$$
w \cdot(-2 \alpha-\beta-\gamma)=w(-2 \alpha-\beta-\gamma)-\sum_{\gamma \in \Phi_{w}} \gamma \geq \alpha_{0} .
$$

Further, arguing as in subsection 5.3 , we conclude that $w$ has to satisfy that $w \alpha=$ $-\alpha_{0}, w \beta<0, w \gamma<0$ and $\ell(w)=3$; but, $\alpha_{0} \in \Phi_{w}$ only if $l(w) \geq n-1$ and we have assumed that $n \geq 6$.

Assume next that $\chi=-2 \alpha-2 \beta$, where $\alpha, \beta \in \Phi^{+}$are distinct. Suppose that there exists a $w \in W$, such that $w \cdot \chi \in X^{+}(T)$ and $w \cdot \chi \neq 0$. Making use of (5.11) again we see that

$$
w(-2 \alpha-2 \beta+\rho)-\rho=\alpha_{0}+\lambda_{0}
$$

for some $\lambda_{0} \in \mathbb{Z}_{+} \Phi^{+}$. In particular, we have

$$
\begin{aligned}
& \langle-2 \alpha-2 \beta+\rho,-2 \alpha-2 \beta+\rho\rangle=\langle w(-2 \alpha-2 \beta+\rho), w(-2 \alpha-2 \beta+\rho)\rangle \\
& =\left\langle\alpha_{0}+\lambda_{0}+\rho, \alpha_{0}+\lambda_{0}+\rho\right\rangle \geq\left\langle\alpha_{0}+\rho, \alpha_{0}+\rho\right\rangle=2 n+\langle\rho, \rho\rangle .
\end{aligned}
$$

It follows that

$$
\langle\alpha, \alpha\rangle+\langle\beta, \beta\rangle+2\langle\alpha, \beta\rangle-\langle\alpha+\beta, \rho\rangle \geq n / 2 .
$$

This can only happen when

$$
n=6,\langle\alpha, \beta\rangle=1,\langle\alpha, \rho\rangle=2,\langle\beta, \rho\rangle=1 .
$$

Here we can assume that ht $\alpha=\langle\alpha, \rho\rangle \geq \operatorname{ht} \beta=\langle\beta, \rho\rangle$.

Suppose that $n=6$ and $\beta=\alpha_{i}, 1 \leq i \leq 5$. Then $\alpha=\alpha_{i}+\alpha_{i+1}$ or $\alpha=\alpha_{i-1}+\alpha_{i}$. In the first case we have that $-2 \alpha-2 \beta=-4 \alpha_{i}-2 \alpha_{i+1}$, and

$$
\begin{cases}\left\langle-4 \alpha_{i}-2 \alpha_{i+1}+\rho,\left(\alpha_{i-1}+\alpha_{i}\right)^{\vee}\right\rangle=0 & \text { if } i \geq 2, \\ \left\langle-4 \alpha_{i}-2 \alpha_{i+1}+\rho,\left(\sum_{j=i}^{i+3} \alpha_{j}\right)^{\vee}\right\rangle=0 & \text { if } i \leq 2 .\end{cases}
$$

In the second case we have that $-2 \alpha-2 \beta=-2 \alpha_{i-1}-4 \alpha_{i}$ and

$$
\begin{cases}\left\langle-2 \alpha_{i-1}-4 \alpha_{i}+\rho,\left(\alpha_{i}+\alpha_{i+1}\right)^{\vee}\right\rangle=0 & \text { if } i \leq 4 \\ \left\langle-2 \alpha_{i-1}-4 \alpha_{i}+\rho,\left(\sum_{j=i-3}^{i} \alpha_{j}\right)^{\vee}\right\rangle=0 & \text { if } i \geq 4\end{cases}
$$

It follows that in these cases $-2 \alpha-2 \beta$ does not contribute to the potential support as $H^{k}\left(X, \mathcal{L}_{-2 \alpha-2 \beta}\right)=0$ for all $k$.

Assume that $\chi=-3 \alpha-\beta$, where $\alpha, \beta \in \Phi^{+}$are distinct roots. Suppose that there exists a $w \in W$, such that $w \cdot \chi \in X^{+}(T)$ and $w \cdot \chi \neq 0$. Arguing as above, we have

$$
9\langle\alpha, \alpha\rangle+\langle\beta, \beta\rangle+6\langle\alpha, \beta\rangle-6\langle\alpha, \rho\rangle-2\langle\beta, \rho\rangle \geq 2 n .
$$

This can happen only if $n \leq 8,\langle\alpha, \beta\rangle=1,3\langle\alpha, \rho\rangle+\langle\beta, \rho\rangle \leq 13-n$, or if $n=6$, $\langle\alpha, \beta\rangle=0$, and $\langle\alpha, \rho\rangle=\langle\beta, \rho\rangle=1$. More precisely, if $n=6$ and $\langle\alpha, \beta\rangle=1$, then the possible values for $(\langle\alpha, \rho\rangle,\langle\beta, \rho\rangle)$ are $(1,2),(1,3),(1,4),(2,1)$; if $n=7$ and $\langle\alpha, \beta\rangle=1$, then the possible values for $(\langle\alpha, \rho\rangle,\langle\beta, \rho\rangle)$ are $(1,2),(1,3)$; and if $n=8$ and $\langle\alpha, \beta\rangle=1$, then $(\langle\alpha, \rho\rangle,\langle\beta, \rho\rangle)=(1,2)$. 
Suppose first that $\langle\alpha, \beta\rangle=1$ and $\alpha=\alpha_{i}, 1 \leq i \leq n-1$. Then $\beta=\sum_{k=i}^{j} \alpha_{k}$ for some $j \geq i+1$ or $\beta=\sum_{k=j}^{i} \alpha_{k}$ for some $j \leq i-1$. In the first case we have $-3 \alpha-\beta=-4 \alpha_{i}-\sum_{k=i+1}^{j} \alpha_{k}$ and

$$
\begin{array}{ll}
\left\langle-3 \alpha-\beta+\rho, \alpha_{j}^{\vee}\right\rangle=0 & \text { if } j \neq i+1, \\
\left\langle-3 \alpha-\beta+\rho,\left(\sum_{k=i}^{i+3} \alpha_{k}\right)^{\vee}\right\rangle=0 & \text { if } j=i+1 \text { and } i \leq n-4, \\
\left\langle-3 \alpha-\beta+\rho,\left(\sum_{k=i-2}^{i} \alpha_{k}\right)^{\vee}\right\rangle=0 & \text { if } j=i+1 \text { and } i \geq 3 ;
\end{array}
$$

in the second case we have $-3 \alpha-\beta=-\sum_{k=j}^{i-1} \alpha_{k}-4 \alpha_{i}$ and

$$
\begin{array}{ll}
\left\langle-3 \alpha-\beta+\rho, \alpha_{j}^{\vee}\right\rangle=0 & \text { if } j \neq i-1, \\
\left\langle-3 \alpha-\beta+\rho,\left(\sum_{k=i}^{i+2} \alpha_{k}\right)^{\vee}\right\rangle=0 & \text { if } j=i-1 \text { and } i \leq n-3, \\
\left\langle-3 \alpha-\beta+\rho,\left(\sum_{k=i-3}^{i} \alpha_{k}\right)^{\vee}\right\rangle=0 & \text { if } j=i-1 \text { and } i \geq 4 .
\end{array}
$$

Thus, in all of these cases $-3 \alpha-\beta$ does not contribute to the potential support.

Suppose next that $n=6,\langle\alpha, \beta\rangle=1,\langle\alpha, \rho\rangle=2$ and $\langle\beta, \rho\rangle=1$. Let us write $\beta=\alpha_{i}$. Then $\alpha=\alpha_{i}+\alpha_{i+1}$ or $\alpha=\alpha_{i-1}+\alpha_{i}$. We have

$$
\begin{array}{ll}
\left\langle-4 \alpha_{i}-3 \alpha_{i+1}+\rho,\left(\sum_{k=i-1}^{i+1} \alpha_{k}\right)^{\vee}\right\rangle=0 & \text { if } i \geq 2, \\
\left\langle-4 \alpha_{i}-3 \alpha_{i+1}+\rho,\left(\sum_{k=i}^{i+3} \alpha_{k}\right)^{\vee}\right\rangle=0 & \text { if } i \leq 2
\end{array}
$$

or

$$
\begin{array}{ll}
\left\langle-3 \alpha_{i-1}-4 \alpha_{i}+\rho,\left(\sum_{k=i-1}^{i+1} \alpha_{k}\right)^{\vee}\right\rangle=0 & \text { if } i \leq 4, \\
\left\langle-3 \alpha_{i-1}-4 \alpha_{i}+\rho,\left(\sum_{k=i-3}^{i} \alpha_{k}\right)^{\vee}\right\rangle=0 & \text { if } i \geq 4 .
\end{array}
$$

Thus, again, in this case $-3 \alpha-\beta$ does not contribute to the potential support.

Suppose now that $n=6,\langle\alpha, \beta\rangle=0,\langle\alpha, \rho\rangle=\langle\beta, \rho\rangle=1$. Let us write $\alpha=\alpha_{i}$ and $\beta=\alpha_{j}$. Then either $j \geq i+2$ or $j \leq i-2$. We have

$$
\begin{array}{ll}
\left\langle-3 \alpha_{i}-\alpha_{j}+\rho,\left(\sum_{k=i}^{i+2} \alpha_{k}\right)^{\vee}\right\rangle=0 & \text { if } j>i+3, \\
\left\langle-3 \alpha_{i}-\alpha_{j}+\rho,\left(\sum_{k=i}^{i+1} \alpha_{k}\right)^{\vee}\right\rangle=0 & \text { if } j=i+2, \\
\left\langle-3 \alpha_{i}-\alpha_{j}+\rho,\left(\sum_{k=i}^{i+3} \alpha_{k}\right)^{\vee}\right\rangle=0 & \text { if } j=i+3, \\
\left\langle-3 \alpha_{i}-\alpha_{j}+\rho,\left(\sum_{k=i-2}^{i} \alpha_{k}\right)^{\vee}\right\rangle=0 & \text { if } j<i-3, \\
\left\langle-3 \alpha_{i}-\alpha_{j}+\rho,\left(\sum_{k=i-1}^{i} \alpha_{k}\right)^{\vee}\right\rangle=0 & \text { if } j=i-2, \\
\left\langle-3 \alpha_{i}-\alpha_{j}+\rho,\left(\sum_{k=i-3}^{i} \alpha_{k}\right)^{\vee}\right\rangle=0 & \text { if } j=i-3 .
\end{array}
$$

Thus, also in this case $-3 \alpha-\beta$ does not contribute to the potential support.

Finally, assume that $\chi=-4 \alpha$. If $\operatorname{ht}(\alpha) \geq 4$, then $\chi$ is a sum of distinct negative roots, so we can assume that ht $(\alpha) \leq 3$. Suppose that $\alpha=\alpha_{i}+\alpha_{i+1}+\alpha_{i+2}$. If $i \leq$ $n-4$, then $\left\langle-4 \alpha+\rho,\left(\sum_{j=i}^{i+3} \alpha_{j}\right)^{\vee}\right\rangle=0$; if $i \geq 2$, then $\left\langle-4 \alpha+\rho,\left(\sum_{j=i-1}^{i+2} \alpha_{j}\right)^{\vee}\right\rangle=0$; thus, $\mathrm{H}^{k}\left(X, \mathcal{L}_{\chi}\right)=0$ for all $k$.

Suppose that $\alpha=\alpha_{i}+\alpha_{i+1}$. If $i \leq n-4$, then $\left\langle-4 \alpha+\rho,\left(\sum_{j=i}^{i+3} \alpha_{j}\right)^{\vee}\right\rangle=0$; if $i \geq 3$, then $\left\langle-4 \alpha+\rho,\left(\sum_{j=i-2}^{i+1} \alpha_{j}\right)^{\vee}\right\rangle=0$; thus $\mathrm{H}^{k}\left(X, \mathcal{L}_{\chi}\right)=0$ for all $k$.

Suppose that $\alpha=\alpha_{i}$. If $i \leq n-4$, then $\left\langle-4 \alpha+\rho,\left(\sum_{j=i}^{i+3} \alpha_{j}\right)^{\vee}\right\rangle=0$; if $i \geq 4$, then $\left\langle-4 \alpha+\rho,\left(\sum_{j=i-3}^{i} \alpha_{j}\right)^{\vee}\right\rangle=0$; thus $\mathrm{H}^{k}\left(X, \mathcal{L}_{\chi}\right)=0$ for all $k$ unless when $n=6$ and $i=3$. In this case we have

$$
-4 \alpha_{3}=\left(s_{5} s_{1} s_{4} s_{2} s_{3}\right) \cdot\left(\alpha_{1}+2 \alpha_{2}+3 \alpha_{3}+2 \alpha_{4}+\alpha_{5}\right) .
$$

This completes the proof of (5.19). 
Consider the short exact sequences

$$
\begin{aligned}
0 \rightarrow \mathfrak{b} \otimes \mathfrak{b}^{\otimes i} \otimes(\mathfrak{g} / \mathfrak{b})^{\otimes(3-i)} & \rightarrow \mathfrak{g} \otimes \mathfrak{b}^{\otimes i} \otimes(\mathfrak{g} / \mathfrak{b})^{\otimes(3-i)} \\
& \rightarrow \mathfrak{g} / \mathfrak{b} \otimes \mathfrak{b}^{\otimes i} \otimes(\mathfrak{g} / \mathfrak{b})^{\otimes(3-i)} \rightarrow 0, i=0,1,2,3 .
\end{aligned}
$$

From the exact sequence with $i=3$ we conclude, making use of (5.8), that

$$
\mathrm{H}^{0}\left(X, \mathfrak{b}^{\otimes 4}\right)=0, \mathrm{H}^{k}\left(X, \mathfrak{b}^{\otimes 4}\right) \cong \mathrm{H}^{k-1}\left(X, \mathfrak{b}^{\otimes 3} \otimes \mathfrak{g} / \mathfrak{b}\right) \text { for } k \geq 4 \text { or } k=1
$$

and we further obtain the exact sequence

$$
\begin{aligned}
0 \rightarrow \mathrm{H}^{1}\left(X, \mathfrak{b}^{\otimes 3} \otimes \mathfrak{g} / \mathfrak{b}\right) & \rightarrow \mathrm{H}^{2}\left(X, \mathfrak{b}^{\otimes 4}\right) \rightarrow \mathfrak{g}^{\oplus 2} \rightarrow \mathrm{H}^{2}\left(X, \mathfrak{b}^{\otimes 3} \otimes \mathfrak{g} / \mathfrak{b}\right) \\
& \rightarrow \mathrm{H}^{3}\left(X, \mathfrak{b}^{\otimes 4}\right) \rightarrow 0 .
\end{aligned}
$$

Using the exact sequence with $i=2$ and making use of (5.17) and (5.8) we get

$$
\mathrm{H}^{0}\left(X, \mathfrak{b}^{\otimes 3} \otimes \mathfrak{g} / \mathfrak{b}\right)=0, \mathrm{H}^{k}\left(X, \mathfrak{b}^{\otimes 3} \otimes \mathfrak{g} / \mathfrak{b}\right) \cong \mathrm{H}^{k-1}\left(X, \mathfrak{b}^{\otimes 2} \otimes(\mathfrak{g} / \mathfrak{b})^{\otimes 2}\right) \text { for } k \geq 3
$$

and we obtain the exact sequence

$$
\begin{aligned}
0 \rightarrow \mathrm{H}^{0}\left(X, \mathfrak{b}^{\otimes 2} \otimes(\mathfrak{g} / \mathfrak{b})^{\otimes 2}\right) & \rightarrow \mathrm{H}^{1}\left(X, \mathfrak{b}^{\otimes 3} \otimes \mathfrak{g} / \mathfrak{b}\right) \rightarrow \mathfrak{g}^{\oplus 2} \oplus(\mathfrak{g} \otimes \mathfrak{g})^{\oplus 2} \\
& \rightarrow \mathrm{H}^{1}\left(X, \mathfrak{b}^{\otimes 2} \otimes(\mathfrak{g} / \mathfrak{b})^{\otimes 2}\right) \rightarrow \mathrm{H}^{2}\left(X, \mathfrak{b}^{\otimes 3} \otimes \mathfrak{g} / \mathfrak{b}\right) \rightarrow 0 .
\end{aligned}
$$

Using the exact sequence with $i=1$ and making use of (5.17) and (5.8) we get

$$
\mathrm{H}^{k}\left(X, \mathfrak{b}^{\otimes 2} \otimes(\mathfrak{g} / \mathfrak{b})^{\otimes 2}\right) \cong \mathrm{H}^{k-1}\left(X, \mathfrak{b} \otimes \mathfrak{g} / \mathfrak{b}^{\otimes 3}\right) \text { for } k \geq 2,
$$

and the exact sequence

$$
\begin{aligned}
0 \rightarrow \mathrm{H}^{0}\left(X, \mathfrak{b}^{\otimes 2} \otimes(\mathfrak{g} / \mathfrak{b})^{\otimes 2}\right) & \rightarrow \mathfrak{g}^{\oplus 2} \oplus(\mathfrak{g} \otimes \mathfrak{g}) \rightarrow \mathrm{H}^{0}\left(X, \mathfrak{b} \otimes \mathfrak{g} / \mathfrak{b}^{\otimes 3}\right) \\
& \rightarrow \mathrm{H}^{1}\left(X, \mathfrak{b}^{\otimes 2} \otimes(\mathfrak{g} / \mathfrak{b})^{\otimes 2}\right) \rightarrow 0 .
\end{aligned}
$$

Using the exact sequence with $i=0$ and making use of (5.17) and (5.8) we get

$$
\mathrm{H}^{k}\left(X, \mathfrak{b} \otimes(\mathfrak{g} / \mathfrak{b})^{\otimes 3}\right) \cong \mathrm{H}^{k-1}\left(X,(\mathfrak{g} / \mathfrak{b})^{\otimes 4}\right), \quad k \geq 2,
$$

and we obtain the exact sequence

$$
\begin{aligned}
0 \rightarrow \mathrm{H}^{0}\left(X, \mathfrak{b} \otimes(\mathfrak{g} / \mathfrak{b})^{\otimes 3}\right) & \rightarrow \mathfrak{g} \otimes \mathrm{H}^{0}\left(X,(\mathfrak{g} / \mathfrak{b})^{\otimes 3}\right) \rightarrow \mathrm{H}^{0}\left(X,(\mathfrak{g} / \mathfrak{b})^{\otimes 4}\right) \\
& \rightarrow \mathrm{H}^{1}\left(X, \mathfrak{b} \otimes(\mathfrak{g} / \mathfrak{b})^{\otimes 3}\right) \rightarrow 0 .
\end{aligned}
$$

It follows that

$$
\mathrm{H}^{k}\left(X, \mathfrak{b}^{\otimes 4}\right)=0, k=0,1 \text { and } \mathrm{H}^{k}\left(X, \mathfrak{b}^{\otimes 4}\right) \cong \mathrm{H}^{k-4}\left(X,(\mathfrak{g} / \mathfrak{b})^{\otimes 4}\right), k \geq 5
$$

and

$$
\begin{aligned}
0 \rightarrow \mathrm{H}^{0}\left(X, \mathfrak{b} \otimes(\mathfrak{g} / \mathfrak{b})^{\otimes 3}\right) & \rightarrow \mathfrak{g} \otimes H^{0}\left(X,(\mathfrak{g} / \mathfrak{b})^{\otimes 3}\right) \\
& \rightarrow \mathrm{H}^{0}\left(X,(\mathfrak{g} / \mathfrak{b})^{\otimes 4}\right) \rightarrow H^{4}\left(X, \mathfrak{b}^{\otimes 4}\right) \rightarrow 0 .
\end{aligned}
$$

As 0 is not in the potential support of $H^{k}\left(X,(\mathfrak{g} / \mathfrak{b})^{\otimes 4}\right)$, using (5.19), (5.20) and (5.21) we see that (5.18) follows. 
5.5. Type $B_{2}$. As the case $r=1$ applies to any type, we consider the case $r=2$ here. We write $\Phi^{+}=\left\{\alpha_{1}, \alpha_{2}, \alpha_{1}+\alpha_{2}, 2 \alpha_{1}+\alpha_{2}\right\}$, where $\left\langle\alpha_{1}, \alpha_{2}^{\vee}\right\rangle=-1$ and $\left\langle\alpha_{2}, \alpha_{1}^{\vee}\right\rangle=-2$.

Just as in subsection 5.2 one checks that:

$$
\operatorname{PSupp}\left(\mathrm{H}^{k}(X, \mathfrak{b} \otimes \mathfrak{b})\right)= \begin{cases}\{0\} & \text { if } k=0,1,3 \\ \left\{0, \alpha_{1}+\alpha_{2}\right\} & \text { if } k=2 \\ \emptyset & \text { if } k \geq 4\end{cases}
$$

Proceeding as in 5.2 we see that:

$$
\mathrm{H}^{k}(X, \mathfrak{b} \otimes \mathfrak{b})= \begin{cases}0 & \text { if } k=0 \text { of } k \geq 3, \\ \mathbb{C} & \text { if } k=1, \\ L\left(\alpha_{1}+\alpha_{2}\right) & \text { if } k=2 .\end{cases}
$$

The only difference to the argument in subsection 5.2 is that the weight $\alpha_{1}+\alpha_{2}$ occurs for $k=2$ only and it occurs precisely once in that case; otherwise the argument is the same.

\section{Results And COUnTEREXAmples}

6.1. The case of $A_{2}$. In this case we have the following:

Theorem 6.1. For $\mathfrak{g}=\mathfrak{s l}_{3}$ the variety $\mathcal{A}_{r}$ has rational singularities, and thus is Cohen-Macaulay, for all $r$.

Proof. According to Lemma 4.3 it suffices to show that

$$
\mathrm{H}^{i}\left(X, \mathfrak{b}^{\otimes r}\right)=0 \text { for all } i \geq r \geq 1 .
$$

Since cohomology vanishes in degrees above the dimension, we only have to consider the cases $r=1,2,3$. These cases are treated in subsections [5.1, 5.2, and 5.3. respectively.

The fact that $\mathcal{A}_{r}$ is Cohen-Macaulay follows; see for example [K, Page 50].

Remark 6.1. By a slight modification of our methods one can show that this result holds for all characteristics above 3 .

6.2. The case of $B_{2}$ and $r=2$. We will show that $\mathcal{A}_{2}$ is not normal in the case of $B_{2}$. For $\mathcal{A}_{2}$ to be normal, the map

$$
\operatorname{Sym}(\mathfrak{g} \oplus \mathfrak{g}) \rightarrow \mathrm{H}^{0}(X, \operatorname{Sym}(\mathfrak{g} / \mathfrak{b} \oplus \mathfrak{g} / \mathfrak{b}))
$$

has to be onto. In particular, this has to hold for $\mathrm{Sym}^{2}$ and hence, by decomposing the $\mathrm{Sym}^{2}$ on both sides, the map

$$
\mathfrak{g} \otimes \mathfrak{g} \rightarrow \mathrm{H}^{0}(X, \mathfrak{g} / \mathfrak{b} \otimes \mathfrak{g} / \mathfrak{b})
$$

has to be onto. Making use of the spectral sequence (4.5), (5.1), and (5.22) we get that

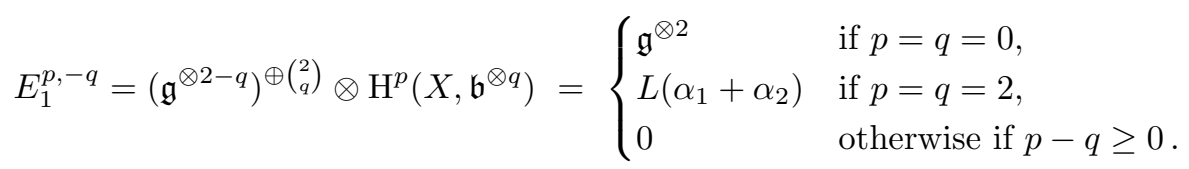

As the term $E_{1}^{2,-2}$ must survive in the spectral sequence, we see that $\mathfrak{g}^{\otimes 2} \rightarrow$ $\mathrm{H}^{0}\left(X,(\mathfrak{g} / \mathfrak{b})^{\otimes 2}\right)$ cannot be onto and so $\mathcal{A}_{2}$ is not normal in this case. 
Remark 6.2. Using the same method as in the previous section, one can show that

$$
\operatorname{PSupp}\left(H^{k}\left(X, \wedge^{3}\left(\mathfrak{b}^{\oplus 2}\right)\right)\right)= \begin{cases}\{0\} & \text { if } k=0,1,4, \\ \left\{0, \alpha_{1}+\alpha_{2}, 2 \alpha_{1}+\alpha_{2}\right\} & \text { if } k=2, \\ \left\{0, \alpha_{1}+\alpha_{2}\right\} & \text { if } k=3 .\end{cases}
$$

In subsection 5.5 we have shown that $H^{i}\left(X, \wedge^{2}\left(\mathfrak{b}^{\oplus 2}\right)\right)=0$ for $i \geq 3$. Making use of the Koszul complex (4.3), we see that $\left.H^{k}\left(X, \wedge^{3}\left(\mathfrak{b}^{\oplus 2}\right)\right)\right) \cong H^{k-3}\left(X, \operatorname{Sym}^{3}\left((\mathfrak{g} / \mathfrak{b})^{\oplus 2}\right)\right)$. Since 0 is not in the potential support of $H^{k}\left(X, \operatorname{Sym}^{3}\left((\mathfrak{g} / \mathfrak{b})^{\oplus 2}\right)\right)$, we conclude that

$$
H^{4}\left(X, \wedge^{3}\left(\mathfrak{b}^{\oplus 2}\right)\right)=H^{1}\left(X, \operatorname{Sym}^{3}\left((\mathfrak{g} / \mathfrak{b})^{\oplus 2}\right)\right)=0 .
$$

Since cohomology vanishes in degrees above the dimension, we see that $H^{i}\left(X, \wedge^{j}\left(\mathfrak{b}^{\oplus 2}\right)\right)=$ 0 for all $j$ and all $i \geq j+1$, which in turn implies that

$$
H^{i}\left(X, \operatorname{Sym}\left((\mathfrak{g} / \mathfrak{b})^{\oplus 2}\right)\right)=0 \text { for all } i \geq 1 .
$$

Thus, we see that the normalization of $\mathcal{A}_{2}$ for $B_{2}$ has rational singularities.

6.3. The case of $A_{3}$ and $r=3$. Making use of the spectral sequence (4.5), (5.1), (5.2), and (5.8) we get that

$$
E_{1}^{p,-q}=\left(\mathfrak{g}^{\otimes 3-q}\right)^{\oplus}\left(\begin{array}{l}
3 \\
q
\end{array}\right) \otimes \mathrm{H}^{p}\left(X, \mathfrak{b}^{\otimes q}\right)= \begin{cases}\mathfrak{g}^{\otimes 3} & \text { if } p=q=0, \\
L\left(\alpha_{1}+2 \alpha_{2}+\alpha_{3}\right) & \text { if } p=q=3, \\
0 & \text { otherwise if } p-q \geq 0 .\end{cases}
$$

As the term $E_{1}^{3,-3}$ must survive in the spectral sequence we see that $\mathfrak{g}^{\otimes 3} \rightarrow$ $\mathrm{H}^{0}\left(X,(\mathfrak{g} / \mathfrak{b})^{\otimes 3}\right)$ cannot be onto and so, arguing as in the case of $B_{2}$ above, we see that $\mathcal{A}_{3}$ is not normal.

Remark 6.3. Similarly to the case of $\mathcal{A}_{2}$ for $B_{2}$, the normalization of $\mathcal{A}_{3}$ for type $A_{3}$ has rational singularities. One proceeds the same way as in that case. In particular, we have

$$
\begin{gathered}
\operatorname{PSupp}\left(H^{k}\left(X, \wedge^{4}\left(\mathfrak{b}^{\oplus 3}\right)\right)\right)= \begin{cases}\{0\} & \text { if } k=5,6, \\
\left\{0, \alpha_{1}+\alpha_{2}+\alpha_{3}, \alpha_{1}+2 \alpha_{2}+\alpha_{3}\right\} & \text { if } k=4, \\
\left\{\begin{array}{l}
0, \alpha_{1}+\alpha_{2}+\alpha_{3}, \alpha_{1}+2 \alpha_{2}+\alpha_{3}, \\
\alpha_{1}+2 \alpha_{2}+2 \alpha_{3}, 2 \alpha_{1}+2 \alpha_{2}+\alpha_{3}
\end{array}\right\} & \text { if } k=3,\end{cases} \\
\operatorname{PSupp}\left(H^{6}\left(X, \wedge^{5}\left(\mathfrak{b}^{\oplus 3}\right)\right)\right)=\left\{\alpha_{1}+\alpha_{2}+\alpha_{3}, 0\right\} .
\end{gathered}
$$

Note that 0 and $\alpha_{1}+\alpha_{2}+\alpha_{3}$ are not in the potential support of $H^{1}\left(X, \operatorname{Sym}^{5}\left((\mathfrak{g} / \mathfrak{b})^{\oplus 3}\right)\right)$.

6.4. The case of $A_{5}$ and $r=4$. Making use of the spectral sequences (4.5), (5.1), (5.2), (5.8), and (5.18) we see that

$$
\begin{aligned}
E_{1}^{p,-q} & =\left(\mathfrak{g}^{\otimes 3-q}\right)^{\oplus\left(\begin{array}{l}
3 \\
q
\end{array}\right) \otimes \mathrm{H}^{p}\left(X, \mathfrak{b}^{\otimes q}\right)} \\
& = \begin{cases}L\left(\alpha_{1}+2 \alpha_{2}+3 \alpha_{3}+2 \alpha_{4}+\alpha_{5}\right) & \text { if } p=5 \text { and } q=4, \\
0 & \text { otherwise if } p-q>0 .\end{cases}
\end{aligned}
$$

As the term $E_{1}^{5,-4}$ must survive in the spectral sequence, we see that $H^{1}\left(X,(\mathfrak{g} / \mathfrak{b})^{\otimes 4}\right)$ $=L\left(\alpha_{1}+2 \alpha_{2}+3 \alpha_{3}+2 \alpha_{4}+\alpha_{5}\right)$. In particular, it is not zero and hence in this case the normalization of $\mathcal{A}_{4}$ does not have rational singularities. 


\section{ACKNOWLEDGEMENTS}

This work was partially performed at the Max Planck Institute for Mathematics in Bonn, the Research Institute of Mathematical Sciences in Kyoto, and the Mathematical Sciences Research Institute in Berkeley. The authors thank these institutions for their hospitality. We also thank Frank Calegari for bringing this question to our attention. Furthermore, we thank the referee for careful reading of the manuscript and for helpful suggestions.

\section{REFERENCES}

[CM] J.-Y. Charbonnel and A. Moreau, Nilpotent bicone and characteristic submodule of a reductive Lie algebra, Transform. Groups 14 (2009), no. 2, 319-360, DOI 10.1007/s00031009-9048-y. MR 2504926

[H] W. H. Hesselink, Cohomology and the resolution of the nilpotent variety, Math. Ann. 223 (1976), no. 3, 249-252. MR0417195

[J] J. C. Jantzen, Representations of algebraic groups, 2nd ed., Mathematical Surveys and Monographs, vol. 107, American Mathematical Society, Providence, RI, 2003. MR2015057

[K] G. Kempf, F. F. Knudsen, D. Mumford, and B. Saint-Donat, Toroidal embeddings. I, Lecture Notes in Mathematics, Vol. 339, Springer-Verlag, Berlin-New York, 1973. MR0335518

[KW1] H. Kraft and N. R. Wallach, Polarizations and nullcone of representations of reductive groups, Symmetry and spaces, Progr. Math., vol. 278, Birkhäuser Boston, Inc., Boston, MA, 2010, pp. 153-167, DOI 10.1007/978-0-8176-4875-6_8. MR2562627

[KW2] H. Kraft and N. R. Wallach, On the nullcone of representations of reductive groups, Pacific J. Math. 224 (2006), no. 1, 119-139, DOI 10.2140/pjm.2006.224.119. MR.2231654

[LMP] M. Losik, P. W. Michor, and V. L. Popov, On polarizations in invariant theory, J. Algebra 301 (2006), no. 1, 406-424, DOI 10.1016/j.jalgebra.2005.07.008. MR2230339

[S] A. Snowden, Singularities of ordinary deformation rings, arXiv:1111.3654.

School of Mathematics and Statistics, University of Melbourne, ViC 3010, Australia - And - Department of Mathematics and Statistics, University of Helsinki, Helsinki, FINLAND

E-mail address: kari.vilonen@unimelb.edu.au

E-mail address: kari.vilonen@helsinki.fi

School of Mathematics and Statistics, University of Melbourne, ViC 3010, Australia - And - Department of Mathematics and Statistics, University of Helsinki, Helsinki, FINLAND

E-mail address: ting.xue@unimelb.edu.au 